\title{
Monitoring Process Variation Using Modified EWMA
}

Aamir Saghir ${ }^{1}$, Muhammad Aslam², ${ }^{*}$ Ali Reza Faraz ${ }^{3 \& 4}$, Liaquat Ahmad $^{5}$, Cedric Heuchenne $^{3}$

${ }^{1}$ Department of Mathematics, Mirpur University of Science and Technology (MUST), Mirpur10250 (AJK), Pakistan. E-mail:aamirstat@yahoo.com

${ }^{2}$ Department of Statistics, Faculty of Science, King Abdulaziz University, Jeddah 21551, Saudi

Arabia.E-mail: aslam_ravian@hotmail.com

${ }^{3}$ HEC Liège, Management School of theUniversity of Liège, Liège 4000, Belgium.

${ }^{4}$ Logistikum, University of Applied Sciences Upper Austria, Steyr 4400, Austria. Email:

alireza.faraz@gmail.com

${ }^{5}$ Department of Statistics and computer Science, UVAS Business School, University of

Veterinary and Animal Sciences, Lahore 54000, Pakistan

E-mail: liaquatahmad@uvas.edu.pk

\begin{abstract}
A new control chart, namely modified EWMA control chart, for monitoring the process variance is introduced in this work by following the recommendations of Khan et $a l .{ }^{15}$. The proposed control chart deduces the existing charts to be its special cases. The necessary coefficients, which are required for the construction of modified EWMA chart, are determined for various choices of sample sizes and smoothing constants. The performance of the proposed modified EWMA is evaluated in terms of its run length (RL) characteristics such as average RL (ARL) and standard deviation of RL (SDRL). The efficiency of the modified EWMA chart is investigated and compared study with some existing control charts. The comparison reveals the superiority of proposal as compared to others control charts in terms of early detection of shift in process variation. The application of the proposal is also demonstrated using a real life dataset.
\end{abstract}

Keywords: Control Charts, Modified statistic, EWMA, run length, Variation, Markov Chain. 
*corresponding author

\section{Introduction}

Control chart is an important tool of statistical process control (SPC) for monitoring the process deviation and improving the quality of the target variable (see, Montgomery ${ }^{1}$ ). Two types of control charts i.e., variable and attribute are commonly used in the literature of the control chart. Variable control charts are used for the numeric characteristics of the interested quality as the height, weight or temperature while the attribute control charts are developed for the non-numeric characteristics of the interested quality as the good/defective items, satisfied/not-satisfied or the small/medium/large items. The variable quality characteristics provide more information about the process as compared to the attribute ${ }^{2}$. Location charts are developed for monitoring of the process average while the dispersion charts are applied for monitoring the process variation. Dispersion charts are considered the speedy chart for the process variation monitoring. The study of the dispersion chart is also important as it is common assumption of the Shewhart average chart to assume that the process standard deviation rests constant (see, Acosta-Mejia et al. ${ }^{3}$ ). The dispersion charts have been explored by many quality control researchers including Lowery et al. ${ }^{4}$.

Exponentially weighted moving average (EWMA) charts was introduced by Roberts ${ }^{5}$ and originally, it was developed for normally distributed data, but today the method has found widespread usage for online monitoring of analytical processes (Abbasi ${ }^{6}$ ), industrial production processes (Lucas and Saccucci ${ }^{7}$ ), public health surveillance (Woodall ${ }^{8}$ ), and other procedures, where the outcomes of the event are obtained sequentially, but not necessarily coming from a normal distribution. An EWMA chart dominates the Shewhart chart in detecting small to moderate shifts in terms of statistical performance. The general form for the two-sided EWMA chart is given in many textbooks, including Montgomery ${ }^{1}$. The EWMA statistic is the exponentially weighted average of the previous as well as the current observations. The typical weight parameter $\lambda$ (ranging from0 to 1), also known as a smoothing parameter, performs well for small values (between 0.05 and 0.25 ) for monitoring the production processes ${ }^{1}$. A lot of work has been done in the literature on designing and studying the performance of EWMA control charts including ${ }^{9-11}$.

The researchers are continuously endeavoring to develop a robust technique for the early detection of the shifted process so that the losses incurred by the manufactured process be avoided. Gan $^{12}$ developed the modified EWMA control chart for the monitoring of the binomial counts. 
Later, Gan ${ }^{13}$ introduced three modified EWMA statistics for detecting smaller shifts in the process for which the Shewhart chart is less effective. Patel and Divecha ${ }^{14}$ proposed a modified EWMA chart for monitoring the process mean when small and abrupt shifts occur in the process. Recently, in order to increase the fast detecting ability of the EWMA statistic, a modified EWMA statistic was introduced by Khan et al. ${ }^{15}$. Unlike the conventional EWMA statistic a second parameter $k$ is introduced in it for quick detection of the small process shifts. It has been observed that the modified EWMA statistic is capable of detecting overall mean shift as compared to the traditional EWMA statistic. Latterly, Schmid ${ }^{16}$ proposed modified EWMA control chart for the monitoring the time series data. Other work on the development of EWMA control charts based on modified EWMA statistics includes Aslam et al. ${ }^{17}$, Khan et al. ${ }^{18}$, Herdiani et al. ${ }^{19}$, Zhang et al. ${ }^{20}$, Lampereia et al. ${ }^{21}$, etc. All these articles concluded that the modified EWMA control chart is more capable of detecting early shift in process parameter under study as compared to the traditional EWMA control chart. Therefore, this article proposes a EWMA control chart using modified EWMA statistic for efficient monitoring of process variation.

The rest of the paper is organized as follow: design of the proposed chart is developed in Section 2. In Section 3, the performance of the proposed chart is given. In Section 4, the designing for the estimation of parameters of the modified EWMA chart is explained. In Section 5, the designing of the out-of-control performance of the proposed chart and its comparison is elaborated. The application of the proposed chart is demonstrated in Section 6. Finally, the conclusions and the future recommendations are given in the Section 7.

\section{Design of the Proposed Chart}

It is assumed that the quality of interest $X_{\mathrm{t}}(t \geq 1)$ follows the normal distribution with mean $\mu$ and variance $\sigma^{2}$. Our interest is only to detect any change in the process dispersion $\delta_{t}^{2}=\sigma_{t}^{2} / \sigma_{0}^{2}$ where $\sigma_{t}^{2}$ is shifted variance and $\sigma_{0}^{2}$ in-control variance. Without loss of generality, it is assumed that $\mu=0$. Define a random variable $Y_{t}=\ln \left(S_{t}^{2} / \sigma_{0}^{2}\right)$, where $S_{t}^{2} / \sigma_{0}^{2}$ is a gamma distributed random variable with parameters $(n-1) / 2$ and $2 \delta_{t}^{2} /(n-1)$. The resulting distribution of $Y_{t}$ is log-gamma distribution and Lawless ${ }^{22}$ showed that it can be approximated by a normal distribution, i.e., $Y_{t} \approx N\left(\mu_{Y}, \sigma_{Y}^{2}\right)$, where

$$
\mu_{Y}=\ln \left(\delta_{t}^{2}\right)-\frac{1}{n-1}-\frac{1}{3(n-1)^{2}}+\frac{2}{15(n-1)^{4}} \quad \text { and } \quad \sigma_{Y}^{2}=\frac{2}{n-1}+\frac{2}{(n-1)^{2}}+\frac{4}{3(n-1)^{3}}-\frac{16}{15(n-1)^{5}} .
$$


Based on this assumption, we propose a control chart using Modified EWMA statistic for monitoring the process variation.

Let a random sample $X_{\mathrm{t} 1}, X_{\mathrm{t} 2}, \ldots, X_{\mathrm{tn}}$ of size $n$ is randomly drawn at time (or subgroup) $t$ and measure $Y_{t}$. The modified EWMA statistic, denoted by $\mathrm{M}_{\mathrm{t}}$, having a smoothing constant $\lambda$ at time $\mathrm{t}$ can be defined as:

$$
\mathrm{M}_{\mathrm{t}}=(1-\lambda) \mathrm{M}_{\mathrm{t}-1}+\lambda \mathrm{Y}_{\mathrm{t}}+\mathrm{k}\left(\mathrm{Y}_{\mathrm{t}}-\mathrm{Y}_{\mathrm{t}-1}\right)
$$

Here, $\mathrm{M}_{0}=0$ and $\mathrm{Y}_{\mathrm{t}}=\ln \left(S_{t}^{2} / \sigma_{0}^{2}\right)$ is log-transformed random variable which is ratio of sample variance to population variance. The smoothing constant in the range of $0.05<\lambda \leq 0.25$ is usually recommended by Montgomery ${ }^{1}$. The constant $-1 \leq k \leq 1$ may be chosen independently of $\lambda$, but in this study the optimal choice for $k$ is $k=-\lambda / 2$, which is derived by Khan et al. ${ }^{15}$. Further note that, when $k=0$, the modified EWMA statistics reduces to Crowder and Hamilton ${ }^{23}$ EWMA statistic and at $k=1$, it reduces to Patel and Divecha ${ }^{14}$ first order auto-correlated EWNMA statistic. After successive substitution, statistic $\mathrm{M}_{t}$ defined in equation (1) can be rewritten as:

$$
\mathrm{M}_{t}=(1-\lambda)^{t} M_{0}+\sum_{i=0}^{t-1}(1-\lambda)^{i}\left[(\lambda+k) Y_{t-i}-k Y_{t-i-1}\right]
$$

The mean and variance of modified EWMA statistic are given as follows

$$
E\left(M_{t}\right)=\mu_{Y} \text { and } V\left(M_{t}\right)=\frac{\left(\lambda+2 \lambda \mathrm{k}+2 \mathrm{k}^{2}\right)}{(2-\lambda)} \sigma_{Y}^{2}
$$

To detect an increase in process variance, the EWMA statistic $Q_{t}=\max \left(M_{t}, 0\right)$ signals an out of control if $\mathrm{Q}_{\mathrm{t}}$ is greater than

$$
U C L=L_{Q} \sqrt{\frac{\left(\lambda+2 \lambda \mathrm{k}+2 \mathrm{k}^{2}\right)}{(2-\lambda)} \sigma_{Y}^{2}}
$$

where $L_{Q}$ can be determined for fixed value of $n$ and to achieve a desired $A R L_{0}$.

Similarly, a decrease in process variance signals an out of control if $Q_{t}=\min \left(M_{t}, 0\right)$ is greater than

$$
L C L=-L_{\dot{Q}} \sqrt{\frac{\left(\lambda+2 \lambda \mathrm{k}+2 \mathrm{k}^{2}\right)}{(2-\lambda)} \sigma_{Y}^{2}}
$$

where $L_{Q}$ is control charting constant and can be determined for fixed value of $n$ to achieve a desired $A R L_{0}$. 
The limits defined in equations (3) and (4) are called one sided control limits of the modified EWMA statistic. However, the two sided control limits for modified EWMA chart can be determine using both statistics $Q_{t}$ and $Q_{t}$. In the rest of this article, we will consider a two sided modified EWMA control chart.

The two sided control limits for the modified EWMA are defined as

$$
\left.\begin{array}{l}
L C L=-L \sqrt{\frac{\left(\lambda+2 \lambda \mathrm{k}+2 \mathrm{k}^{2}\right)}{(2-\lambda)} \sigma_{Y}^{2}} \\
U C L=L \sqrt{\frac{\left(\lambda+2 \lambda \mathrm{k}+2 \mathrm{k}^{2}\right)}{(2-\lambda)} \sigma_{Y}^{2}}
\end{array}\right\},
$$

where $L=L_{Q}=L_{\dot{Q}}$ is control chart coefficient need to be determined for a fixed value of $n, \lambda, k$ and desire in-control $A R L_{0}$. The proposed modified EWMA chart is based on two constants $\lambda$ and $k$. The proposed chart is the extension of the existing control charts. The proposed control chart reduces to control chart proposed by Crowder and Hamilton ${ }^{23}$ when $k=0$.

The proposed modified EWMA control chart will signal if $\mathrm{UCL}<\mathrm{M}_{t}<L C L$, otherwise the process is declared to be work under in-control scenario. The probability of signal can be find as:

$$
\text { Prob. of signal }=\operatorname{Pr}\left(U C L<M_{t}<L C L \mid \delta_{t}^{2}\right)
$$

\section{The Performance Evaluation}

The average run length (ARL), which is the mean of RL distribution, is the most important and widely used measure to evaluate the performance of control charts. The ARL is defined as the average number of subgroups until an out-of-control signal is raised. In case, the process is in control, the ARL should be sufficiently large to avoid too many false alarms while for the out-ofcontrol process; the ARL should be sufficiently small for rapid detection of shift.

$$
\mathrm{ARL}_{0}=1 / \operatorname{Pr}\left(L C L<M_{t}<U C L \mid \delta_{t}^{2}=1\right)
$$

and

$$
\mathrm{ARL}_{1}=\frac{1}{\text { Prob.of Signal }}
$$

The accurate measure of ARL has been thoroughly discussed by Hussain et al. ${ }^{24}$. ARL for the EWMA chart have been discussed by Chananet et al. ${ }^{25}$ and Li et al. ${ }^{26}$

The equation (8) and (9) can be solved easily if the probability density function of modified EWMA statistic $M_{t}$ is known. The exact distribution of modified EWMA statistic defined in 
equation (1) is more explicit and up to our best knowledge is not available in the literature. Therefore, the run length characteristic of the proposed control chart can be investigated through Monte Carlo simulation method. For this purpose, the following algorithm in $R$ package is developed and run length characteristic are obtained for various choices of parameters.

\section{Algorithms}

The following algorithms has been used in $R$ to find the results of this study.

\section{Algorithm 1: Determination of control charting constant $(L)$}

Step 1. Sample of size $n$ is drawn from Normal distribution $(0,1)$, without loss of generality, and $Y_{i}=\ln \left(S_{i}^{2} / \sigma_{0}^{2}\right)$ is calculated.

Step 2. Using the value of $Y_{i}$ obtained in step 1, modified EWMA statistic $M_{\mathrm{t}}$ is calculated for a specified value of $\lambda$ and $k$. This process is repeated up to 10,000 times and at the end of this step we have 10,000 modified EWMA statistic's.

Step 3. The control charting constant $L$ is determined to satisfying the in-control condition of $\operatorname{Pr}\left(L C L<M_{t}<U C L\right)=\alpha$.

Step 4. The above steps 1 to 3 are repeated 1,000 times and at the end of this step we have 1000 $L$ values for specific choice of $n, \lambda$ and $k$.

Step 5. Finally, the average value of $L$ is chosen for further analysis.

\section{Algorithm 2: Determination of run length characteristics}

Step 1. Control limits of the modified EWMA chart are constructed using the charting constant determined in above algorithm for specific choice of $n, \lambda$ and $k$.

Step2. Sample of size $n$ is drawn from Normal distribution $\left(0, \sigma_{1}^{2}\right)$, and $Y_{i}=\ln \left(S_{i}^{2} / \sigma_{1}^{2}\right)$ is calculated.

Step 3. Using the value of $Y_{i}$ obtained in step 2, modified EWMA statistic $M_{\mathrm{t}}$ is calculated.

Step 4. The probability of signal using the equation (7) and run length using the equation (9) are calculated.

Step 4. The above steps 1 to 4 are repeated 10,000 times and at the end of this step we have 10,000 average run length values. 
Step 5. Finally, the average and standard deviation of 10,000 average run length values. These ARL and SDRL are used

\section{Designing for the estimation of parameters of the modified EWMA chart}

The traditional design procedure for selecting the parameters of EWMA charts based on only the average run length (ARL) may lead to high probability of false out-of-control signal for some types of EWMA charts as discussed by Chan and Zhang ${ }^{27}$. They also recommended the procedure for selecting the parameters based on the average run length and standard deviation of the run length (SDRL) with the constraint that $S D R L \leq A R L$ when the process is in-control and out-of-control. Using the algorithm 1, a Monte Carlo simulation approach with 10,000 iterations is used to approximate the RL distribution of the proposed modified EWMA chart. Note that $\mathrm{Kim}^{28}$ and Schaffer and $\mathrm{Kim}^{29}$ indicated that 5,000 replications are sufficient to estimate the ARL to an acceptable level of precision in many control chart settings. The zero-state or initial-state ARL's are computed in this study. The control charting constant $L$ is determined in such a way that the two one-sided charts produces equal individual $A R L_{0}$ so that the overall $A R L_{0}$ of the two-sided chart is approximately equal to 200. The values of $L$, for a fixed value of $n, \lambda, k$ and $A R L_{0}=200$, are given in Table 1.

Table 1: The control chart multiplier $L$ for various values of $n, \lambda$ and $k$ to achieve the incontrol $A R L_{0}=200$.

\begin{tabular}{|l|c|c|c|c|c|c|}
\hline \multirow{2}{*}{$k$} & \multirow{2}{*}{$n$} & \multicolumn{7}{|c|}{$\lambda$} \\
\cline { 2 - 7 } & & 0.05 & 0.10 & 0.15 & 0.20 & 0.50 \\
\hline \multirow{4}{*}{0} & 5 & 1.194 & 1.570 & 1.761 & 1.971 & 2.009 \\
\cline { 2 - 7 } & 8 & 1.285 & 1.616 & 1.835 & 2.010 & 2.025 \\
\cline { 2 - 7 } & 10 & 1.360 & 1.672 & 1.870 & 2.027 & 2.052 \\
\cline { 2 - 7 } & 15 & 1.473 & 1.758 & 1.933 & 2.057 & 2.088 \\
\hline \multirow{4}{*}{$-\lambda / 2$} & 5 & 0.975 & 1.269 & 1.469 & 1.635 & 2.107 \\
\cline { 2 - 7 } & 8 & 1.121 & 1.386 & 1.550 & 1.681 & 2.164 \\
\cline { 2 - 7 } & 10 & 1.207 & 1.467 & 1.620 & 1.735 & 2.274 \\
\cline { 2 - 7 } & 15 & 1.340 & 1.590 & 1.725 & 1.821 & 2.367 \\
\hline \multirow{4}{*}{1} & 5 & 4.122 & 3.824 & 3.640 & 3.515 & 3.296 \\
\cline { 2 - 7 } & 8 & 4.082 & 3.803 & 3.624 & 3.481 & 3.164 \\
\cline { 2 - 7 } & 10 & 4.045 & 3.785 & 3.601 & 3.465 & 3.105 \\
\cline { 2 - 7 } & 15 & 4.001 & 3.762 & 3.585 & 3.440 & 3.055 \\
\hline
\end{tabular}


From Table 1, it can be seen that:

a. the control charting constant depend on $k, n$ and $\lambda$ for a fixed $A R L_{0}$.

b. the values of $L$ decreases with increase in sample size when others parameters are fixed.

c. increase in smoothing weight increase the value of $L$ for a fixed $k$ and $n$ except the value of $k=1$.

\section{Out-of-control Performance}

Suppose that the process goes out-of-control with respect to variance and $\sigma_{1}^{2}=\rho . \sigma_{0}^{2}$ be the new process variance with $\rho$ time change in $\sigma_{0}^{2}$. Under the assumption that the data follows a Normal distribution with mean 0 (without loss of generality) and variance $\sigma_{1}^{2}$, the probability of signal given in (7) can be redefined as;

$$
\begin{aligned}
& P_{\text {out }}=\operatorname{Pr}\left(\mathrm{M}_{\mathrm{t}}>U C L \mid \sigma_{1}^{2}\right) \operatorname{orP}\left(\mathrm{M}_{\mathrm{t}}<L C L \mid \sigma_{1}^{2}\right) \\
& P_{\text {out }}=\operatorname{Pr}\left(\mathrm{M}_{\mathrm{t}}>U C L \mid \sigma_{1}^{2}\right)+P\left(\mathrm{M}_{\mathrm{t}}<L C L \mid \sigma_{1}^{2}\right) \\
& P_{\text {out }}=1-F_{\mathrm{M}_{\mathrm{t}}}\left(U C L \mid \rho \sigma_{0}^{2}\right)+F_{\mathrm{M}_{\mathrm{t}}}\left(L C L \mid \rho \sigma_{0}^{2}\right)
\end{aligned}
$$

where $F_{\mathrm{M}_{\mathrm{t}}}($.$) be the cumulative density function of \mathrm{M}_{\mathrm{t}}$.

The exact probability density function of modified $\mathrm{M}_{\mathrm{t}}$ is explicitly complicated and is not available in close form. Therefore, again we have used simulation procedure to determine the empirical CDF of $\mathrm{M}_{\mathrm{t}}$. The above algorithm 2 is used and $P_{\text {out }}$ determined for various choices of $n, k, \lambda$ and $\rho$.

Table 2 through 5 the values of ARL and SDRL have been estimated using the above mentioned equations. The zero state run length performance of the modified EWMA (MEWMA) control chart for the sample of size 5 and 10 , the smoothing constant $\lambda=0.05$ and 0.10 and control constant $k=0,-\lambda / 2$ and 1 are given for different shift levels 0.50 to 2.00 . It can be observed from these tables that SDRL is always smaller than ARL for all the process settings. A control chart scheme is considered as a better one if it has smaller ARL values, so the proposed chart is studied at different choice of $k$ to identify the suitable value of $k$.

Table 2: Zero state run length performance of the MEWMA chart at $n=5$ and $\lambda=0.05$

\begin{tabular}{|c|l|l|l|l|l|l|}
\hline \multirow{2}{*}{$\rho$} & \multicolumn{3}{|c|}{$k=0$} & \multicolumn{2}{l|}{$k=-\lambda / 2$} & \multicolumn{2}{l|}{$k=1$} \\
\cline { 2 - 6 } & ARL & SDRL & ARL & SDRL & ARL & SDRL \\
\hline
\end{tabular}




\begin{tabular}{|c|c|c|c|c|c|c|}
\hline 0.50 & 4.567 & 2.345 & 2.287 & 1.154 & 7.314 & 5.670 \\
\hline 0.60 & 8.995 & 7.956 & 4.933 & 3.005 & 16.161 & 14.223 \\
\hline 0.70 & 19.104 & 14.234 & 15.424 & 13.256 & 29.717 & 26.745 \\
\hline 0.80 & 40.097 & 31.658 & 29.671 & 23.228 & 55.216 & 52.325 \\
\hline 0.90 & 85.707 & 65.323 & 77.093 & 68.098 & 104.284 & 93.2715 \\
\hline 1.00 & 200.747 & 188.776 & 201.071 & 189.751 & 200.890 & 192.356 \\
\hline 1.10 & 84.345 & 75.563 & 71.508 & 60.005 & 96.345 & 85.456 \\
\hline 1.20 & 33.756 & 25.097 & 26.504 & 20.534 & 61.345 & 55.445 \\
\hline 1.30 & 18.677 & 11.876 & 15.841 & 10.054 & 41.223 & 34.449 \\
\hline 1.40 & 13.087 & 7.082 & 11.170 & 9.113 & 25.334 & 19.777 \\
\hline 1.50 & 10.542 & 5.667 & 9.083 & 4.264 & 18.544 & 14.332 \\
\hline 2.00 & 6.793 & 3.045 & 5.005 & 1.813 & 11.223 & 7.556 \\
\hline
\end{tabular}

Table 3: Zero state run length performance of the MEWMA chart at $n=5$ and $\lambda=0.10$

\begin{tabular}{|c|c|c|c|c|c|c|}
\hline \multirow{2}{*}{$\rho$} & \multicolumn{2}{|c|}{$k=0$} & \multicolumn{2}{c|}{$k=-\lambda / 2$} & \multicolumn{2}{c|}{$k=1$} \\
\cline { 2 - 7 } & ARL & SDRL & ARL & SDRL & ARL & SDRL \\
\hline 0.50 & 3.987 & 1.567 & 2.106 & 0.986 & 5.445 & 3.099 \\
\hline 0.60 & 9.177 & 5.470 & 4.086 & 2.896 & 17.189 & 11.234 \\
\hline 0.70 & 21.123 & 17.209 & 11.037 & 9.556 & 38.334 & 26.223 \\
\hline 0.80 & 60.223 & 51.890 & 35.888 & 30.022 & 90.990 & 80.224 \\
\hline 0.90 & 110.445 & 98.990 & 102.924 & 91.990 & 130.776 & 122.113 \\
\hline 1.00 & 201.005 & 192.113 & 200.628 & 192.556 & 200.888 & 189.445 \\
\hline 1.10 & 85.456 & 76.003 & 76.998 & 69.977 & 101.12 & 94.332 \\
\hline 1.20 & 42.220 & 35.344 & 32.755 & 27.564 & 72.339 & 63.224 \\
\hline 1.30 & 25.539 & 19.223 & 18.706 & 12.867 & 48.228 & 39.002 \\
\hline
\end{tabular}




\begin{tabular}{|c|c|c|c|c|c|c|}
\hline 1.40 & 16.435 & 10.066 & 12.532 & 7.725 & 31.097 & 21.091 \\
\hline 1.50 & 13.112 & 7.199 & 9.976 & 5.378 & 25.489 & 13.223 \\
\hline 2.00 & 8.509 & 3.244 & 5.020 & 2.114 & 14.559 & 8.223 \\
\hline
\end{tabular}

Table 4: Zero state run length performance of the MEWMA chart at $n=10$ and $\lambda=0.05$

\begin{tabular}{|c|c|c|c|c|c|c|}
\hline \multirow{2}{*}{$\rho$} & \multicolumn{2}{|c|}{$k=0$} & \multicolumn{2}{c|}{$k=-\lambda / 2$} & \multicolumn{2}{c|}{$k=1$} \\
\cline { 2 - 7 } & ARL & SDRL & ARL & SDRL & ARL & SDRL \\
\hline 0.50 & 4.223 & 2.005 & 1.850 & 0.653 & 7.889 & 4.889 \\
\hline 0.60 & 8.778 & 5.334 & 3.672 & 2.417 & 15.224 & 9.225 \\
\hline 0.70 & 29.334 & 21.234 & 16.871 & 10.077 & 54.334 & 43.532 \\
\hline 0.80 & 156.445 & 143.223 & 144.05 & 130.091 & 165.223 & 155.229 \\
\hline 0.90 & 177.445 & 170.089 & 159.009 & 141.223 & 186.056 & 156.228 \\
\hline 1.00 & 200.998 & 192.345 & 200.934 & 196.058 & 201.279 & 191.287 \\
\hline 1.10 & 35.445 & 29.770 & 29.116 & 22.572 & 71.890 & 60.011 \\
\hline 1.20 & 21.224 & 17.556 & 12.535 & 6.758 & 43.245 & 37.220 \\
\hline 1.30 & 14.283 & 10.990 & 8.333 & 3.508 & 28.346 & 20.078 \\
\hline 1.40 & 8.724 & 5.332 & 6.609 & 2.255 & 15.489 & 9.881 \\
\hline 1.50 & 7.665 & 3.020 & 5.661 & 1.709 & 12.114 & 6.546 \\
\hline 2.00 & 6.670 & 1.776 & 4.243 & 0.8042 & 10.134 & 3.078 \\
\hline
\end{tabular}

Table 5: Zero state run length performance of the MEWMA chart at $n=10$ and $\lambda=0.10$.

\begin{tabular}{|c|c|c|c|c|c|c|}
\hline \multirow{2}{*}{$\rho$} & \multicolumn{2}{|c|}{$k=0$} & \multicolumn{2}{c|}{$k=-\lambda / 2$} & \multicolumn{2}{c|}{$k=1$} \\
\cline { 2 - 7 } & ARL & SDRL & ARL & SDRL & ARL & SDRL \\
\hline 0.50 & 2.980 & 1.107 & 1.676 & 0.524 & 4.309 & 3.002 \\
\hline
\end{tabular}




\begin{tabular}{|c|c|c|c|c|c|c|}
\hline 0.60 & 4.329 & 2.128 & 2.766 & 1.455 & 7.998 & 4.978 \\
\hline 0.70 & 15.224 & 9.298 & 8.433 & 6.766 & 33.234 & 21.223 \\
\hline 0.80 & 78.334 & 64.297 & 52.211 & 48.550 & 108.334 & 98.768 \\
\hline 0.90 & 142.223 & 136.556 & 105.671 & 99.224 & 167.889 & 151.244 \\
\hline 1.00 & 200.005 & 192.334 & 200.211 & 191.456 & 199.098 & 189.223 \\
\hline 1.10 & 42.099 & 36.445 & 31.366 & 25.446 & 68.345 & 59.456 \\
\hline 1.20 & 20.334 & 14.234 & 13.033 & 7.953 & 42.998 & 36.890 \\
\hline 1.30 & 13.229 & 9.078 & 8.232 & 4.005 & 28.334 & 21.003 \\
\hline 1.40 & 11.870 & 7.345 & 6.356 & 2.493 & 20.889 & 14.334 \\
\hline 1.50 & 8.889 & 4.329 & 5.366 & 1.809 & 15.754 & 9.329 \\
\hline 2.00 & 5.768 & 2.248 & 3.912 & 0.825 & 9.356 & 4.982 \\
\hline
\end{tabular}

Table 2 through 5 shows that:

1. The proposed chart tends to become Crowder and Hamilton ${ }^{23}$ chart when the control $k=0$.

2. The proposed control chart performs better for the choice of $k=-\lambda / 2$ than any other value of $k$ ( 0 or 1 considered here). For example when a sample of $n=5$ and $\lambda=0.05$ is used then a shift of 0.70 is detected by an average samples of 19.104 for the existing chart $(k=0)$ while the proposed control chart detects the same shift in average of samples of $15.424(k=-\lambda / 2)$. This shows that the proposed chart is $23 \%$ better in detecting this shift. The same performance can be seen for other process settings.

3. The modified EWMA control chart performs better than the case when $k=1$ ( as considered by Patel and Divecha ${ }^{14}$ ), for example when a sample of $n=5$ and $\lambda=0.05$ is used then a shift of 0.70 is detected by an average samples of 29.717 for the existing chart while the proposed control chart detects the same shift in average of samples of 15.424. This shows that the proposed chart is $93 \%$ better in detecting this shift. The same performance can be seen for other process settings. 
The comparative efficiency of the proposed chart has also been shown on the graph. It can be seen in the Figures 1 through 3. The proposed chart shows better efficiency in early detecting the outof-control process for all process settings at different shift levels.
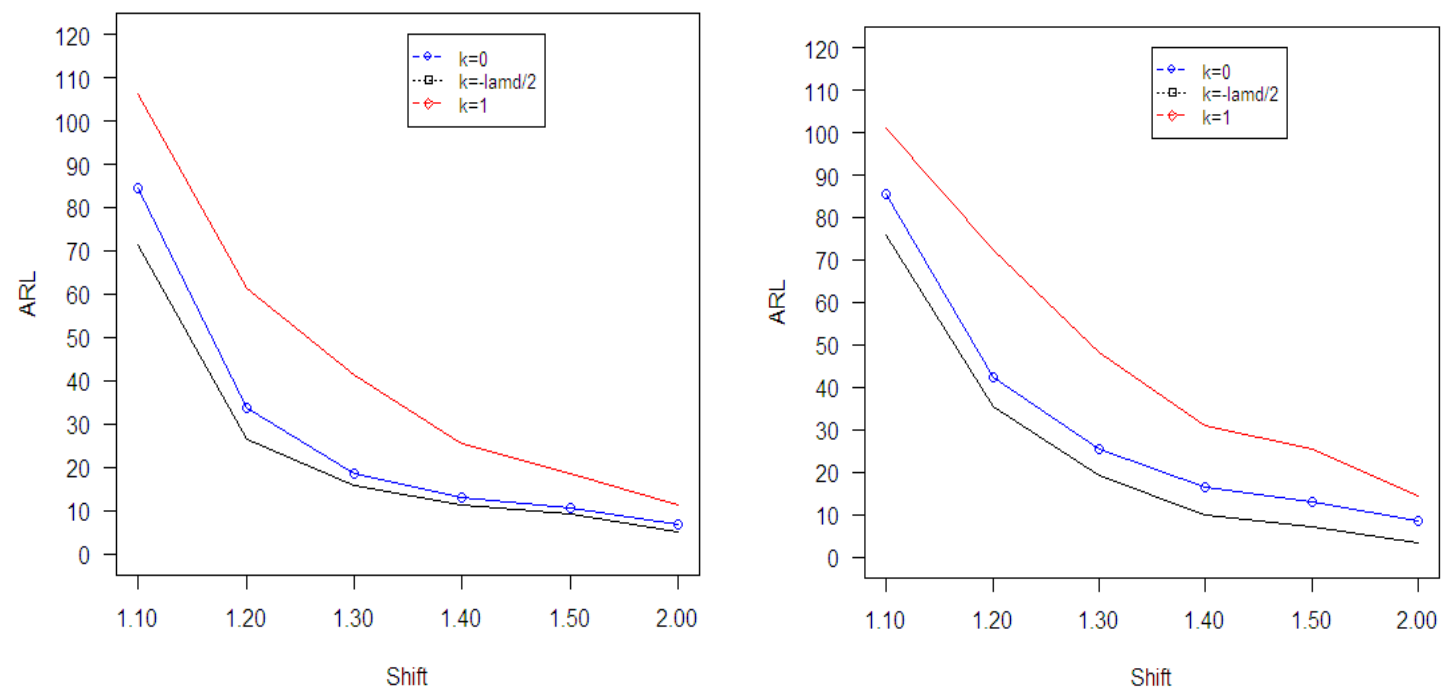

Figure 1. ARL curves of the modified EWMA chart at (a) $\lambda=0.05$ (b) $\lambda=0.10$ for $n=5$ when process variance increases.

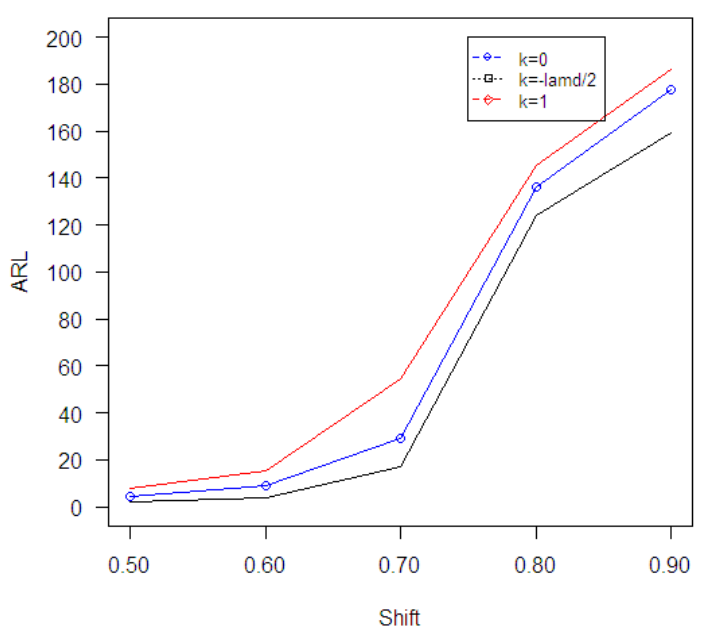

(a)

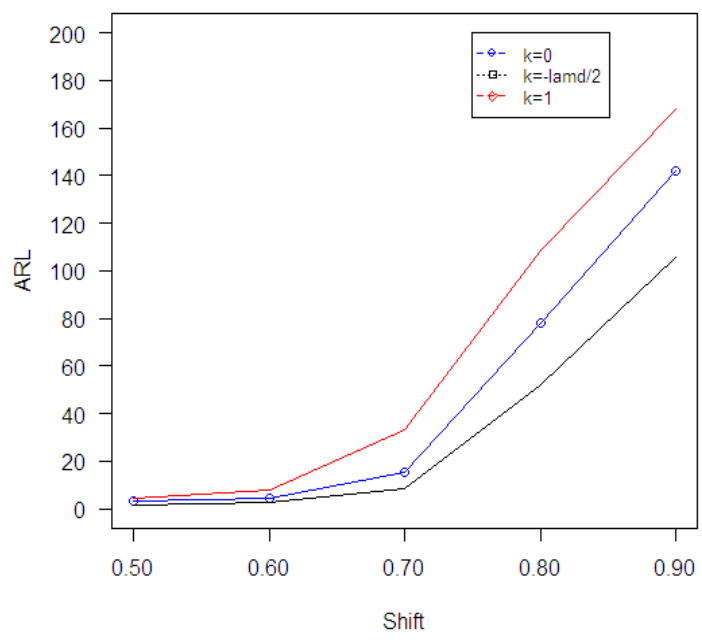

(b) 
Figure 2. ARL curves of the modified EWMA chart at (a) $\lambda=0.05$ (b) $\lambda=0.10$ for $n=10$ when process variance decreases.

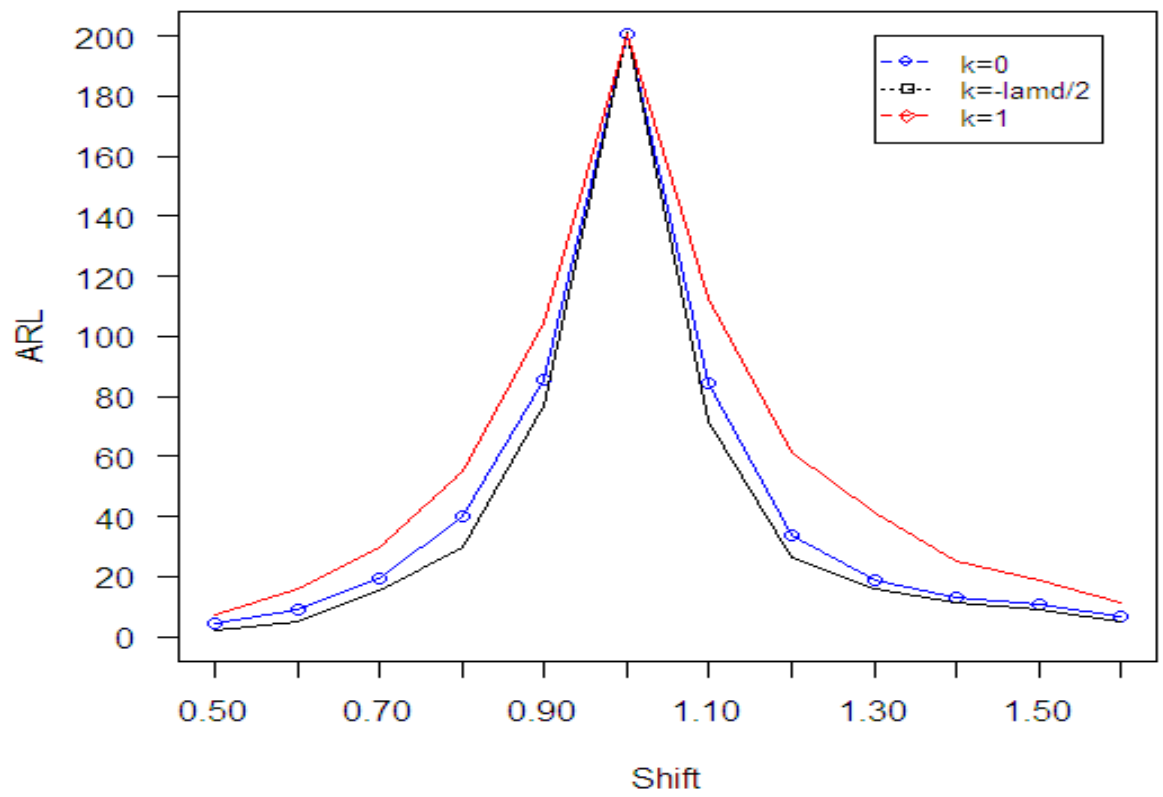

Figure 3. ARL curves of the modified EWMA chart for $n=5$ and $\lambda=0.05$ for up and down shifts in variance.

\subsection{Comparison with other EWMA charts}

Further, the efficiency of the proposed modified EWMA variance chart is compared with the recently proposed charts by Huwang et al. ${ }^{30}$ and Zou and Tsung ${ }^{31}$. Fixing the in-control average run length of two charts, the out-of-control average run length is measured for both charts at various values of $\rho$ as efficiency measure. The results of out-of-control ARL are provided here in table 6 and table 7 for $A R L_{0}$. However, the results can be determined for any other value.

Table 6 and Table 7 reveals that the proposed modified EWMA variance chart perform better than the charts of Huwang et al. ${ }^{30}$ and Zou and Tsung ${ }^{31}$. The efficiency of the proposed chart in terms of producing smaller values of out-of-control ARL increases with the increase in sample size. The proposed chart is better than Huwang et al. ${ }^{30}$ control chart for small shift in the process which is desirable in the industry. 
Table 6: Zero state run length performance of the proposed EWMA with Zou and Tsung ${ }^{31}$ chart at $A R L_{0}=370.00$.

\begin{tabular}{|c|r|r|r|r|r|r|}
\hline \multirow{3}{*}{$\rho$} & \multicolumn{6}{|c|}{$\lambda=0.05$} \\
\cline { 2 - 7 } & \multicolumn{2}{|c|}{ Modified EWMA chart at $k=-\lambda / 2$} & \multicolumn{2}{l|}{$\begin{array}{l}\text { Zou and Tsung } \\
\text { chart }\end{array}$} \\
\cline { 2 - 7 } & \multicolumn{2}{|c|}{$n=5$} & \multicolumn{2}{c|}{$n=10$} & \\
\cline { 2 - 7 } & \multicolumn{1}{|c|}{ ARL } & SDRL & ARL & SDRL & ARL & SDRL \\
\hline 1.00 & 369.092 & 350.220 & 368.995 & 347.400 & 370.067 & 355.505 \\
\hline 1.10 & 123.346 & 114.45 & 115.334 & 99.122 & 123.055 & 115.098 \\
\hline 1.20 & 57.045 & 38.980 & 48.766 & 23.334 & 58.098 & 39.955 \\
\hline 1.30 & 32.322 & 21.908 & 27.698 & 15.332 & 33.889 & 25.660 \\
\hline 1.40 & 21.900 & 12.898 & 17.443 & 9.089 & 23.657 & 14.230 \\
\hline 1.50 & 17.334 & 6.890 & 14.190 & 4.556 & 18.909 & 7.905 \\
\hline 2.00 & 6.113 & 4.234 & 4.909 & 3.245 & 7.129 & 5.220 \\
\hline
\end{tabular}

Table 7: Zero state run length performance of the proposed EWMA with Huwang et al. ${ }^{30}$ chart at $A R L_{0}=200.00$.

\begin{tabular}{|c|c|c|c|c|c|c|}
\hline \multirow{4}{*}{$\rho$} & \multicolumn{6}{|c|}{$\lambda=0.05$} \\
\hline & \multicolumn{4}{|c|}{ Modified EWMA chart at $k=-\lambda / 2$} & \multirow{2}{*}{\multicolumn{2}{|c|}{ Huwang et al..$^{30}$ char }} \\
\hline & \multicolumn{2}{|c|}{$n=5$} & \multicolumn{2}{|c|}{$n=10$} & & \\
\hline & ARL & SDRL & ARL & SDRL & ARL & SDRL \\
\hline 0.50 & 2.287 & 1.154 & 1.850 & 0.653 & 6.531 & 4.898 \\
\hline 0.60 & 4.933 & 3.005 & 3.672 & 2.417 & 9.940 & 7.045 \\
\hline 0.70 & 15.424 & 13.256 & 16.871 & 10.077 & 16.925 & 12.233 \\
\hline 0.80 & 29.671 & 23.228 & 144.05 & 130.091 & 34.042 & 27.520 \\
\hline 0.90 & 77.093 & 68.098 & 159.009 & 141.223 & 91.410 & 78.586 \\
\hline 1.00 & 201.071 & 189.751 & 200.934 & 196.058 & 199.000 & 189.660 \\
\hline 1.10 & 71.508 & 60.005 & 29.116 & 22.572 & 103.011 & 90.105 \\
\hline 1.20 & 26.504 & 20.534 & 12.535 & 6.758 & 49.082 & 38.552 \\
\hline
\end{tabular}




\begin{tabular}{|l|r|r|r|r|r|r|}
\hline 1.30 & 15.841 & 10.054 & 8.333 & 3.508 & 29.062 & 21.253 \\
\hline 1.40 & 11.170 & 9.113 & 6.609 & 2.255 & 20.360 & 14.558 \\
\hline 1.50 & 9.083 & 4.264 & 5.661 & 1.709 & 15.402 & 10.998 \\
\hline 2.00 & 5.005 & 1.813 & 4.243 & 0.8042 & 6.931 & 4.045 \\
\hline
\end{tabular}

\section{Illustrative Example}

The application of the proposed modified EWMA chart is investigated in this section using a real dataset given by Jones-Farmer et al. ${ }^{32}$. The data consist of patients waiting time (in minutes) for a colonoscopy procedure. Jones-Farmer et al. ${ }^{32}$ shown that the process is in-control with respect to dispersion parameter. In order to highlight the ability of the modified EWMA structure to detect changes in the process dispersion parameter, we have introduced contaminations in the original data by multiplying the last 6 observations by 3 similar to Abbasi and Miller ${ }^{33}$. Then, the Crowder and Hamilton ${ }^{23}$ chart, named as $\mathrm{CH}$ chart, and the proposed modified EWMA chart are constructed using $\lambda=0.10$ and $k=-\frac{\lambda}{2}$ in figure 4 below.

Figure 4:Modified EWMA Control chart for patients data at lambda $=0.10$

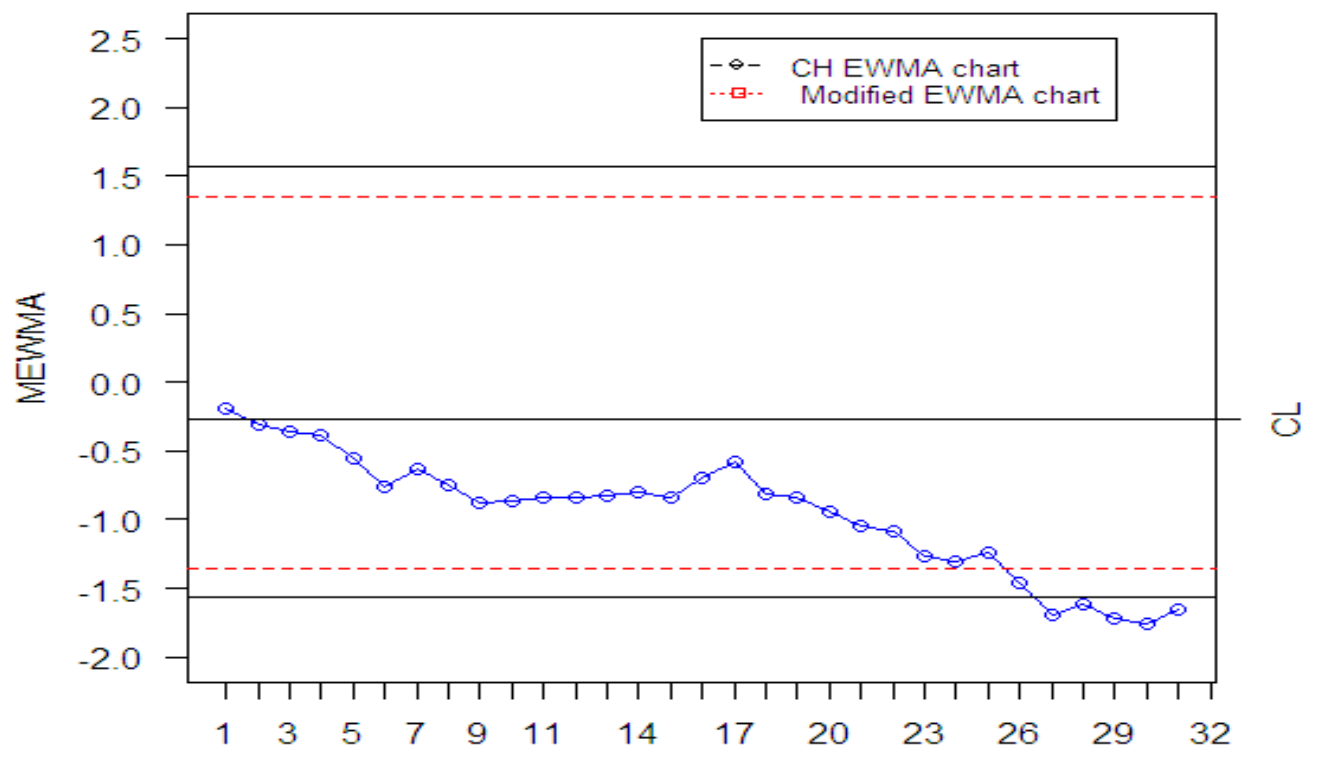

Batch Number 
It is obvious from Figures 4 that the $\mathrm{CH}$ chart detects out-of-control signals at five sample points, whereas the proposed modified EWMA chart detects all the six problem points. Hence, it is concluded that the modified EWMA chart is more efficient than the CH EWMA chart to detect shift in dispersion more quickly.

\section{Conclusions and Recommendations}

In this article, a modified EWMA control chart is proposed for efficient monitoring of the process dispersion following the work of Khan et al. ${ }^{15}$. The control charting constant $L$, which is necessarily required for the construction of the proposed control chart, is determined using Monte Carlo simulation. The performance of the proposed modified EWMA control chart is measured in terms of run length characteristics such as ARL and SDRL for several process dispersion level settings using simulation. Tables of the ARL and the SDRL are generated for different shifts levels, different smoothing constant values and for different control constant values. The proposed control chart has been compared with two existing charts. It has been observed that the proposed chart is efficient in quick detection of the out-of-control process as compared to existing control charts for monitoring process dispersion. These results are very helpful for the practitioners and researchers for monitoring the process dispersion. Further work along the direction to develop modified EWMA control charts for the monitoring multivariate process dispersion and attribute process can be done and authors are currently working on this.

\section{REFERENCES}

1. Montgomery DC. Introduction to Statistical Quality Control (7th edn). John Wiley: New York, 2012.

2. Balamurali S, Jun CH. Repetitive Group Sampling Procedure for Variables Inspection. Journal of Applied Statistics 2006; 33 (3): 327-338.

3. Acosta-Mejia CA, Pignatiello JA, Rao BV. A comparison of control charting procedures for monitoring process dispersion. IIE Transactions 1999; 31 (6): 569-579.

4. Lowry CA, Champ CW, Woodall WH. The performance of control charts for monitoring process variation. Communications in Statistics-Simulation and Computation 1995; 24 (2): 409-437.

5. Roberts SW. A comparison of some control chart procedures. Technimetrics 1966; 8: 411430.

6. Abbasi SA. On the performance of EWMA chart in presence of two components of measurement error. Quality Engineering 2010; 22(3):199-213. 
7. Lucas JM, Saccucci MS. The exponentially weighted moving average control schemes: properties and enhancements (with discussion). Technimetrics 1990; 32:1-12.

8. Woodall WH. The use of control charts in health-care and public-health surveillance. Journal of Quality Technology 2006; 38(2):89-104.

9. Crowder SV. A Simple Method for Studying Run Length Distributions of Exponentially Weighted Moving Average Charts. Technometrics 1987; 29: 401-407

10. Crowder SV. Design of Exponentially Weighted Moving Average Schemes. Journal of Quality Technology 1989; 21 (3): 155-162.

11. Lucas JM, Saccucci MS. Exponentially Weighted Moving Average Control Schemes: Properties and Enhancements. Technometrics 1990; 32 (1): 1-12.

12. Gan F. Monitoring observations generated from a binomial distribution using modified exponentially weighted moving average control chart. Journal of Statistical Computation and Simulation 1990; 37 (1-2): 45-60.

13. Gan F. Monitoring Poisson observations using modified exponentially weighted moving average control charts. Communications in Statistics-Simulation and Computation 1993; 19 (1): 103-124.

14. Patel AK, Divecha J. Modified exponentially weighted moving average (EWMA) control chart for an analytical process data. Journal of Chemical Engineering and Materials Science 2011; 2 (1): 12-20.

15. Khan N, Aslam M, Jun C. H. Design of a control chart using a modified EWMA statistic. Quality and Reliability Engineering International 2017; 33 (5): 1095-1104.

16. Schmid W. On EWMA charts for time series. In Frontiers in statistical quality control, Springer: 1997; 115-137.

17. Aslam M, Saghir A, Ahmad L, Jun C-H, Hussain J. A control chart for COM-Poisson distribution using a modified EWMA statistic. Journal of Statistical Computation and Simulation 2017; 87(18): 3491-3502.

18. Khan N, Yasmin T, Aslam M, Jun C-H. On the performance of modified EWMA charts using resampling schemes. Operations Research and Decisions 2018; 3: 29-43.

19. Herdiani ET, Fandrilla G, Sunusi N. Modified Exponential Weighted Moving Average (EWMA) Control Chart on Autocorrelation Data. Journal of Physics: Conference Series 2018; 979 (1): 012097.

20. Zhang G, Li N, Li S. A modified Multivariate EWMA Control Chart for Monitoring process Small Shifts. Proceedings of 2011 International Conference on Modelling, Identification and Control, Shanghai, China, June 26-29, 2011.

21. Lampreia SPGF, Requeijo JFG, Dias JAM, Vairinhos VM, Barbosa PIS. Condition monitoring based on modified CUSUM and EWMA control charts. Journal of Quality in Maintenance Engineering 2018; 24 (1): 119-132.

22. Lawless, JF. Statistical Models and Methods for lifetime data. John Wiley and Sons: New Jersey, 2003. 
23. Crowder S, Hamilton M. An EWMA for monitoring a process standard deviation. Journal of Quality Technology 1992; 24 (1): 12-21.

24. Hussain S, Song L, Ahmad S, Riaz M. On Auxiliary Information Based Improved EWMA Median Control Charts. Scientia Iranica 2018; 25 (2): 954-982

25. Chananet C, Sukparungsee S, Areepong Y. The ARL of EWMA Chart for Monitoring ZINB Model Using Markov Chain Approach. International Journal of Applied Physics and Mathematics 2014; 4 (4): 236-249.

26. Li ZH, Zou CL, Gong Z, Wang ZJ. The computation of average run length and average time to signal: an overview. Journal of Statistical Computation and Simulation 2014; 84 (8): 17791802.

27. Chan LK, Zhang J. Cumulative sum control charts for covariance matrix. Statistica Sincia 2001; 11(3): 767-790.

28. Kim MJ. Number of replications required in control chart Monte Carlo simulation studies. Ph.D. dissertation, University of Northern Colorado, 2005.

29. Schaffer JR, Kim MJ. Number of replications required in control chart Monte Carlo simulation studies. Communications in Statistics-Simulation and Computation 2007; 36 (5): 1075-1087.

30. Huwang L, Huang CJ, Wang YHT. New EWMA Control Charts for Monitoring Process Dispersion. Computational Statistics and Data Analysis 2010; 54: 2328-2342.

31. Zou C, Tsung E. Likelihood ratio based distribution free EWMA control charts. Journal of Quality Technology 2010; 42 (2): 174-196.

32. Jones-Farmer LA, Jordan A, Champ CW. Distribution-free phase I charts for subgroup location. Journal of Quality Technology 2009; 41(3): 304-316.

33. Abbasi SA, Miller A. On proper choice of variability control chart for Normal and Nonnormal Processes. Quality and Reliability Engineering International 2012; 28: 279-296. 